\title{
Plasmonic Modes and Optical Properties of Gold and Silver Ellipsoidal Nanoparticles by the Discrete Dipole Approximation
}

\author{
Mohammed Alsawafta, Mamoun Wahbeh, and Vo-Van Truong \\ Department of Physics, Concordia University, Montréal, QC, Canada H4B 1R6 \\ Correspondence should be addressed to Vo-Van Truong, tvovan@alcor.concordia.ca
}

Received 17 November 2011; Revised 26 December 2011; Accepted 3 January 2012

Academic Editor: Haibo Zeng

Copyright (C) 2012 Mohammed Alsawafta et al. This is an open access article distributed under the Creative Commons Attribution License, which permits unrestricted use, distribution, and reproduction in any medium, provided the original work is properly cited.

\begin{abstract}
The discrete dipole approximation (DDA) is used to model the absorption efficiency of isolated gold (Au) and silver (Ag) ellipsoidal nanoparticles. The characteristics of the plasmonic bands of those nanostructures depend strongly on the size and orientation of the particles in both the lab and target frames. At specific rotation and incident angles, the desired plasmonic mode can be excited. The result of the simulation shows the possibility of excitation of three plasmonic modes-one longitudinal mode (LM) and two transverse modes (TM) - corresponding to the redistribution of the polarization charges along each principal axis. At oblique incidence of the incoming light, both the Au LM and a hybrid Au TM are observed whereas three more distinct plasmonic modes can be found in the case of the Ag particle. The effect of length distribution on the characteristics of the plasmonic bands is also examined for the three principal axes. The band position of the plasmonic bands associated with the electronic oscillation along each principal axis is found to vary linearly with the axis length. The linear variation of the band position of the LM is steeper as compared with the one found for the other modes.
\end{abstract}

\section{Introduction}

The localized surface plasmon resonance (LSPR) is the electronic oscillatory motion in the conduction band of the metallic nanostructure $[1,2]$. The unique characteristics of such fluctuations originate from the confined spatial distribution of the polarization charges over the surface of the nanostructure [3]. Controlling the size and the shape of the nanoparticles will result in changing the negative/positive charge separation and hence tailoring the frequency and the intensity of the LSPR in the visible and the infrared region [4-6]. Technical applications of metal nanoparticles normally require incorporating an assembly of those nanostructures of different size distributions, and thus understanding the optical behavior of an isolated nanoparticle and the effect of the coupling between the LSPR of nearby particles is required [7-9]. Enhanced capabilities of recent nanofabrication techniques to fabricate and arrange the metallic nanoparticles of different sizes and shapes indeed make them highly attractive in many technical applications [10-22]. Due to the high-order symmetry of spherical nanoparticles, their optical response only exhibits a single PM [23, 24]. In the case of particles with different symmetry axis, more than one PM is observed [25-28] and among the most interesting nanostructures is the ellipsoidal nanoparticle. Due to its 3-fold symmetry, it exhibits both longitudinal and transverse plasmon modes. The oscillatory shift of the negative electron cloud relative to the positive core along each principal axe results in three plasmonic modes (PM), one longitudinal mode (LM), and two transverse modes (TM). The characteristics of each band depend on the orientation of the particle in both the lab and the target frames as well as the length distribution of each axis. The effect of this latter parameter on the extinction coefficient of gold oblate and prolate spheroidal nanoparticles arranged in two dimensional arrays has been studied experimentally [29], and the linear dependency of the peak position of the PM with respect to the length of the corresponding principal axis has been noted. The experimental results were then compared to the ones calculated by the quasistatic approximation (QSA) of the first order (dipole mode). Simulations by a finite-difference time domain (FDTD) 
method were also done but the effect of the target orientation on the possibility of excitation of all the PMs has not been investigated. Another theoretical treatment has been proposed to study the PMs of the ellipsoidal particle equally in the framework of the QSA complemented by the inclusion of higher orders of multipolar oscillations in order to find an analytical expression for the plasmon frequency [30]. On the other hand, Kalkbrenner et al. [31] succeeded in rotating a single gold ellipsoidal nanoparticle attached to the tip of a glass fiber mounted on a stage of a scanning near-field optical microscope (SNOM). Due to the threefold symmetry of the ellipsoidal particle, three PMs were observed individually at a distinct combination of the polarization angle and the rotation angle of the tip in the incident light.

To better appreciate the behavior of particles of this specific and quite interesting shape, the current work aims at studying in a comprehensive way the optical properties of an oriented isolated nanoellipsoidal particle both for the gold $(\mathrm{Au})$ and silver $(\mathrm{Ag})$ cases since these two materials in nanoparticle form exhibit most interesting selective absorption in the visible and near-infrared range. It would thus be of great interest to find whether there exist combinations of the rotation and orientation angles that can allow the simultaneous excitation of all PMs for $\mathrm{Au}$ and $\mathrm{Ag}$ nanoellipsoids. To this end the effect of different parameters on the optical response of the Au and Ag nanoparticles will be discussed. These parameters include the orientation of the target in both the lab and the target frames and the sensitivity of the band position of the PM to the length distribution of each principal axis. To achieve this goal, the DDA [32-37] is employed to calculate the absorption coefficient for both $\mathrm{Au}$ and Ag ellipsoidal nanoparticles. The result found will be useful to optimize the particle properties for applications such as in the plasmonic photovoltaic field $[13,14]$ and in biosensing [21]. As an example, due to the high absorbance of the incident light at different wavelengths in the UV-Vis region, incorporating those nanostructures in the plasmonic solar cell will enhance effectively photoelectrons generation and hence the energy conversion efficiency.

This paper is organized as follows: in Section 2, we will discuss briefly the basic idea of the computational tool DDA, followed by a presentation of the target geometry, the corresponding structural parameters, and the relative orientation of the nanoparticle with respect to the incident electromagnetic field. Section 3 will include the results and discussion, which is divided into two subsections. The first subsection concerns the discussion on the modeled absorption spectra of an isolated nanoellipsoid and the effect of the relative orientation on the excitation of different LSPR modes. Comparison will be made between an $\mathrm{Au}$ particle and an Ag particle of the same size. The second subsection will examine the effect of the axis length distribution on the particle optical properties.

\section{Discrete Dipole Approximation (DDA)}

DDA is one of the well-known computational tools to mimic the optical response of the nanostructure due to the interaction of the target under investigation with the incident electromagnetic waves. To model the morphology of the target, it is required to represent the nanoparticles with an assembly of 3D-induced dipoles. The number of dipoles should be large and in the order of $10^{4}$ to model the precise shape of the target, and requires the interdipole separation to be smaller than the incident wavelength and any structural parameter. The idea of the DDA was first introduced to study the optical response of molecular aggregates $[34,35]$. The retardation effect was not included in the first application of the approximation method. Later, this effect was introduced in the DDA to study the interstellar dust grains [38]. The code of DDA was as well developed to calculate the scattering and the absorption properties of the particles [38]. The formalism of the method was improved by incorporating a correction for radiative reaction and the anisotropic dielectric function [32]. An algorithm called complex-conjugate gradient (CCG) method was then introduced to evaluate the polarization iteratively and use the fast fourier transform (FFT) to solve the matrix-vector multiplications involved in the iteration method (CCG) [33]. The description of the mathematical formulation of the DDA is out of the scope of this paper, but more details can be found in the references cited. Based on the interaction between the induced dipoles, the optical response of the metallic nanostructures can be calculated. The outputs of the DDA are the extinction, absorption and scattering cross-sections of the nanostructure normalized to its geometrical cross section. In this study, the output of the DDA is the absorption cross-section of the nanostructure normalized to its geometrical cross-section, which yields the corresponding efficiencies Q $\mathrm{abs}$. The open-source Fortran-90 software package (DDSCAT 7.1) was used to calculate the absorption cross-section.

\section{Target Geometry and the Target Orientation}

The geometry of the target under investigation is a quadric surface where the morphology is characterized by three semiprincipal axes. The structural parameters of the ellipsoidal nanoparticles are represented by two semi-minor $(2 b$ and $2 c$ ) axes oriented along the $y$ and $z$ axes, respectively, and a semimajor $2(a)$ axis perpendicular to the $y z$ plane. According to the relative length of the three principal axes, the types of the ellipsoidal particles are classified into oblate spheroids $(a=b>c)$, prolate spheroids $(a=b<c)$, and scalene ellipsoids $(a>b>c)$. The latter case is considered in this study as illustrated in Figure 1(a). The effective radius of the equivolume sphere for the ellipsoidal nanoparticles is given by $r_{\mathrm{eff}}=(a * b * c)^{1 / 3}$. The corresponding aspect ratio (A.R) is defined as the ratio of the longest axis to the shortest axis $(a / c)$.

It is assumed that the incident radiation is linearly polarized in the $y$-direction ( $\mathrm{p}$-polarized) and propagates along the $x$-direction. The orientation of the target in the lab frame is achieved by rotating the major axis with respect to the propagation direction $(k)$ by an angle $\theta$ as shown in Figure 1(a). At oblique angles, the incident electric field has two components: one parallel to the $a$-axis and the other one oriented along the $y z$ plan. The electric field of the 


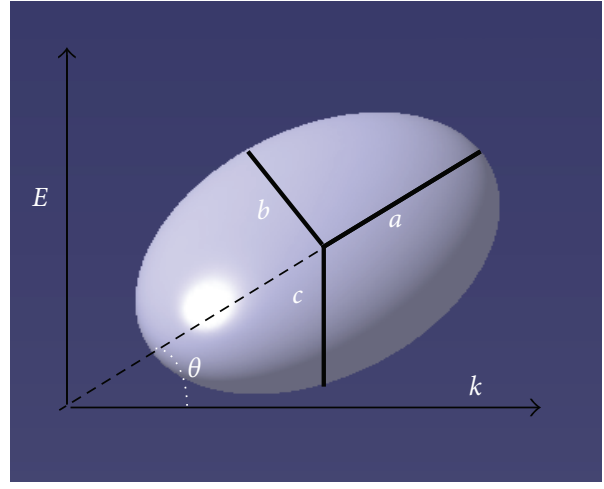

(a)

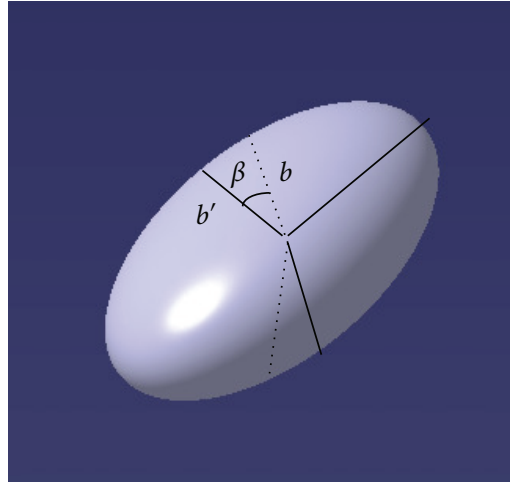

(b)

Figure 1: (a) The geometrical parameters of the ellipsoidal nanoparticle and the orientation of the particle in the lab frame around the $x$-axis with angle $\theta$, (b) the rotation of the nanoellipsoid in the target frame around the $a$-axis with angle $\beta$.

s-polarized light has one component perpendicular to the major axis of the ellipsoid at any angle of incidence, and no information, therefore, is reported on the excitation of all the plasmonic modes. In the case of unpolarized light, the absorption spectrum is calculated as an average over the two polarizations directions, and the spectrum exhibits all LSPR modes.

The absorption spectrum exhibits different dipolar plasmonic modes when the electric field has a component along each principal axis. When the ellipsoidal particle is rotated in the target frame around the main axis by an angle $\beta$ as shown in Figure 1(b), two TMs are observed corresponding to the oscillations of the induced polarization charges, respectively, along the $b$-axis and $c$-axis.

\section{Results and Discussion}

All the DDA calculations presented here refer to air as the surrounding material where the ellipsoidal nanoparticle is embedded. The orientation of the nanoparticle under investigation relative to the direction of the propagation of the incident p-polarized light determines the type of the excited PM. The angle of rotation $(\theta)$ governs the probability of excitation of the LM. The $\beta$ angle plays an important role for the observation of both TMs. On the other hand, all the possible PMs can be found at a distinct combination between the two angles. Those parameters will be addressed in detail in Section 4.1. Section 4.2 will concern the dependency of the band position of the PM on the length distribution and will be presented separately for the excited plasmonic band along each axis.

4.1. Effect of Orientation on the Optical Response of the Ellipsoidal Nanoparticles. The excitation of the desired plasmonic band is achieved at distinct combination between the incident angle and the rotation angle. In multifold symmetry particles, the oscillations of the polarization charges along a certain direction determine the type of the LSPR bands. The redistribution of the charges along the axis of different length changes the separation of the driven electron cloud relative to the positive cores and result in tailoring the intensity and the band position of the LSPR modes.

The effect of the rotation and the orientation of both the metallic ellipsoidal particles is investigated with the electric field oriented along each of the three axes. Three plasmonic bands are reported due to the oscillation of the charges along each one of them. When the incident angle is chosen to be $90^{\circ}$, the major axis is aligned parallel to the incident electric field resulting in the excitation of the LM. Whatever the rotation angle is, no other plasmonic band is observed due to the absence of the induced charges along the other semiaxes. At normal incidence, the nanoellipsoidal particle can be rotated in such a way that the electric field has one component along either the $b$-axis or the $c$-axis, or along both of them. When $\theta=0^{\circ}$ and $\beta=0^{\circ}$ and $90^{\circ}$, the TM along $b$-axis $(b$-TM) and $c$-axis $(c$-TM) is excited, respectively, as shown in Figure 2. The excitation of the three distinct PMs of a single ellipsoidal nanoparticle is consistent with experimental data reported earlier [31]. The most intense plasmonic band corresponds to the LM while the less intense one is for the $c$-TM (for clarity, data in Figure 2 are shown after normalization). The difference in the absorption amplitude would be attributed to the difference in the charge separation (the axis length).

As well, the observed red shift in the LM as compared to the corresponding one in the TMs could be related to the decreasing in the restoring force (the columbic interaction) due to increase of charges separation along the longer axis.

Although the plasma frequencies for both $\mathrm{Au}$ and $\mathrm{Ag}$ would be comparable, the corresponding LSPR mode of the ellipsoidal nanoparticle with the same size occurs at different wavelengths. The deviation originates from the additional contribution of the interband electronic transitions to the dielectric function. The resonance frequency of the LSPR ( $\left.W_{\mathrm{LSPR}}\right)[39]$ is given by

$$
w_{\mathrm{LSPR}}=\frac{w_{p}}{\sqrt{2 \varepsilon_{m}+\chi+1}},
$$

where $w_{P}, \varepsilon_{m}$, and $\chi$ are the plasma frequency of the bulk metal, the dielectric function of the host material where 


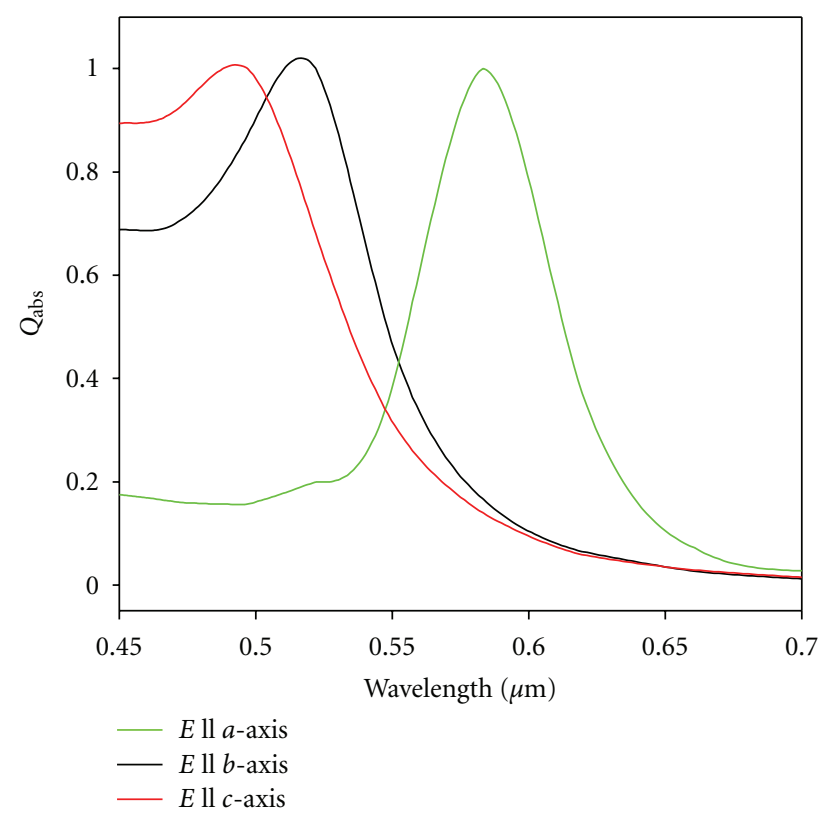

(a)

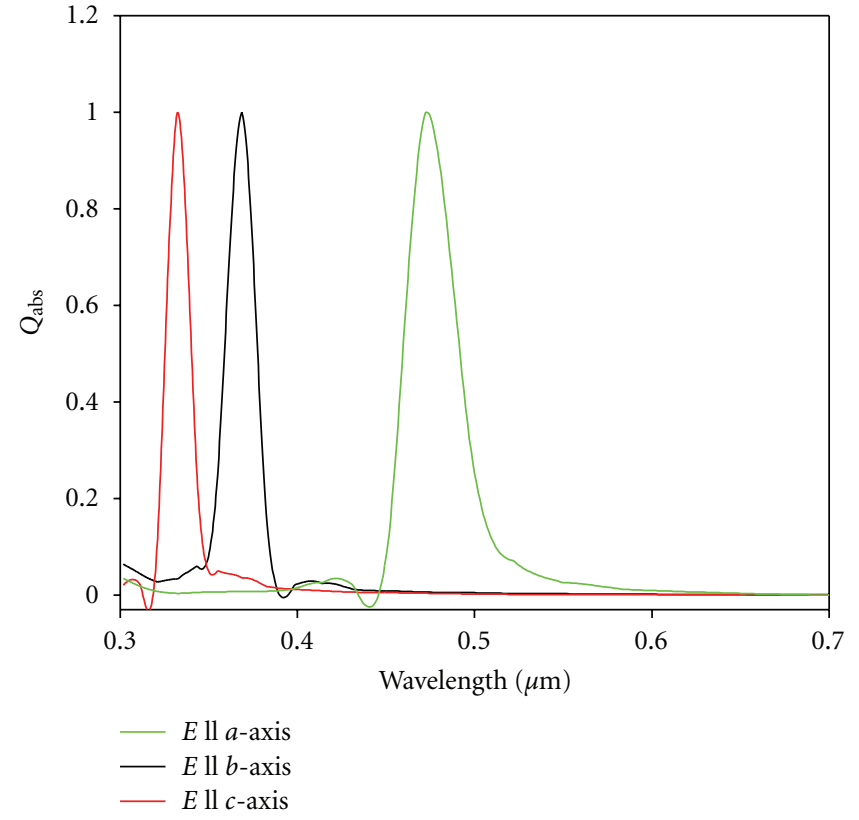

(b)

FIgURE 2: The normalized absorption spectra as a function of the incident wavelength for (a) Au and (b) Ag ellipsoidal nanoparticle. In both cases, the principal axis of the particle is aligned parallel to the incident electric field. The corresponding structural parameters are $2 a$ $=40 \mathrm{~nm}, 2 b=20 \mathrm{~nm}$, and $2 c=10 \mathrm{~nm}$.

the nanoparticles are embedded, and the interband susceptibility, respectively. In the case of the Au nanoparticle, the resonance occurs at higher wavelengths as compared to the one for Ag because $\chi$ - $\mathrm{Au}>\chi$ - $\mathrm{Ag}$.

The full width at half maximum (FWHM) can be compared between the different LSPR bands for both metallic particles. It can be seen that the LM has the larger value among the modes; the Ag-TMs have a comparable width while the Au-b-axis-TM is broadened as compared to the corresponding $c$-axis-TM.

The value of FWHM for the noble metals [39] is given by

$$
\mathrm{FWHM}=\gamma \sqrt{1+\frac{2 \chi_{2} w_{p} /\left(1+\chi_{1}+2 \varepsilon_{m}\right) \sqrt{1+2 \varepsilon_{m}}}{\gamma}},
$$

where $\gamma, \chi_{1}$, and $\chi_{2}$ are, respectively, the damping constant, the real part and the imaginary part of the interband susceptibility. For Ag, at the resonance frequency, because of the small value of $\chi_{2}$ ( $\chi_{2}$ approaches zero), the square root of the last equation is about unity; therefore the value of the bandwidth is equal to $\gamma$ as described in the free electron model. In the case of $\mathrm{Au}$, the imaginary part of the interband transition contributes more to the bulk dielectric function as compared to Ag. Therefore, the square root is larger than unity, and hence the corresponding band width is larger than $\gamma$. This would explain why the plasmonic mode of the $\mathrm{Au}$ nanoparticle is broadened as compared to the one for Ag.

In the case of the $\mathrm{Au}$ ellipsoidal particle, when the excitation of the LM is not possible, and the rotation angle is between 0 and $90^{\circ}$, apparently only a single TM is observed.
In reality the band positions of the TMs are very close to each other, making them indistinguishable, resulting in the excitation of a single broadened band that we would label as a hybrid TM as illustrated in Figure 3(a). The plasmonic bands which correspond to the multifold symmetry are well separated in the case of the Ag particle, showing the excitation of more than a single band (Figure $3(\mathrm{~b})$ ). When $15^{\circ}<\beta<75^{\circ}$, the intensity of the Au-TM has contributions from both $b$-TM and $c$-TM. The amplitude of the hybrid $\mathrm{TM}$ is decreased with $\beta$. Decreasing the value of $\beta$ results in increasing the amplitude of the excited electric field along the $b$-axis. This enhances the absorption amplitude and shifts the TM to a lower energy. The energy difference of the $\mathrm{Ag}$-TMs is larger compared with the calculated one for $\mathrm{Au}$ and it resulted in well-separated modes. No change in the band position of $\mathrm{Ag}$ modes is observed due to change in the rotation angle. Previous observations of the dependency of the absorption amplitude on $\beta$ are still valid for the Ag-TMs.

The LM can be excited in the presence of the TMs. To demonstrate this, $\beta$ has been chosen to be $60^{\circ}$ while the incident angle is changed uniformly in steps of $30^{\circ}$ between the two extreme values of $0^{\circ}$ and $90^{\circ}$ as shown in Figure 4. The absorption spectrum of the Au ellipsoidal particle is characterized by the presence of the most intense plasmonic LM band and the hybrid TM band. The intensity of the latter band is decreased dramatically with the incident angle, while the LM intensity is directly proportional to the incident angle. At $\theta=90^{\circ}$, the incident electric field is perfectly aligned with the major axis which results in a maximum absorption for the LM. At the other extreme of 


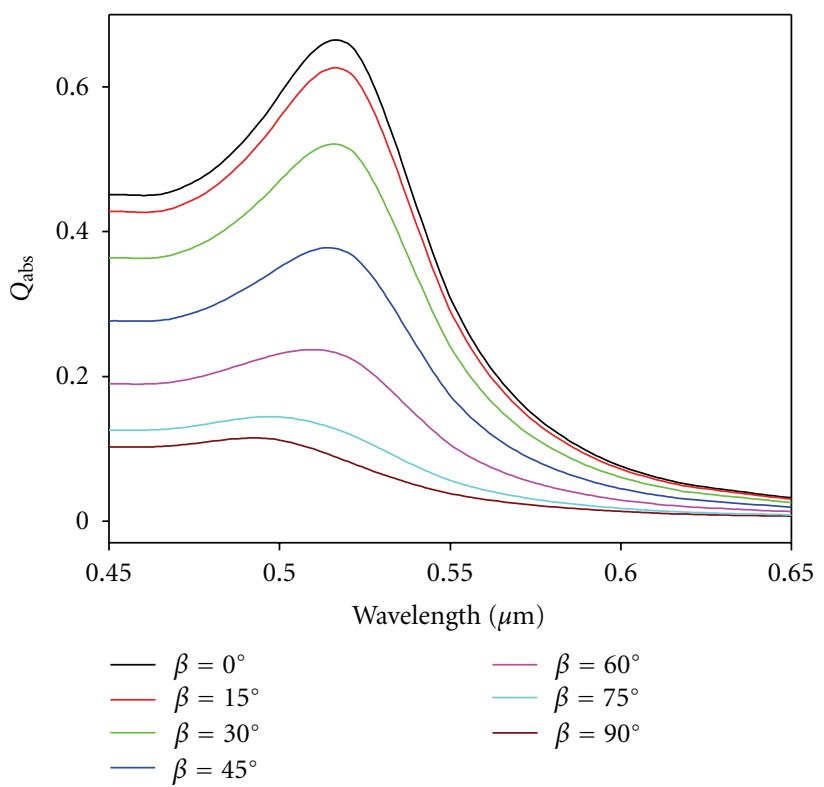

(a)

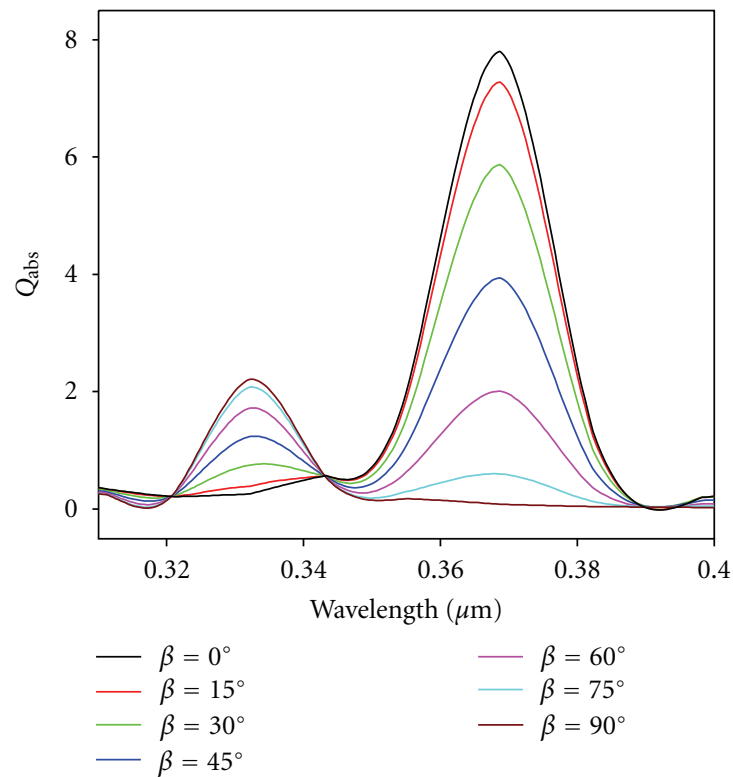

(b)

Figure 3: The dependency of the absorption efficiency on the rotation angle for (a) Au and (b) Ag ellipsoidal nanoparticles. The corresponding structural parameters are $2 a=40 \mathrm{~nm}, 2 b=20 \mathrm{~nm}$ and $2 c=10 \mathrm{~nm}$.

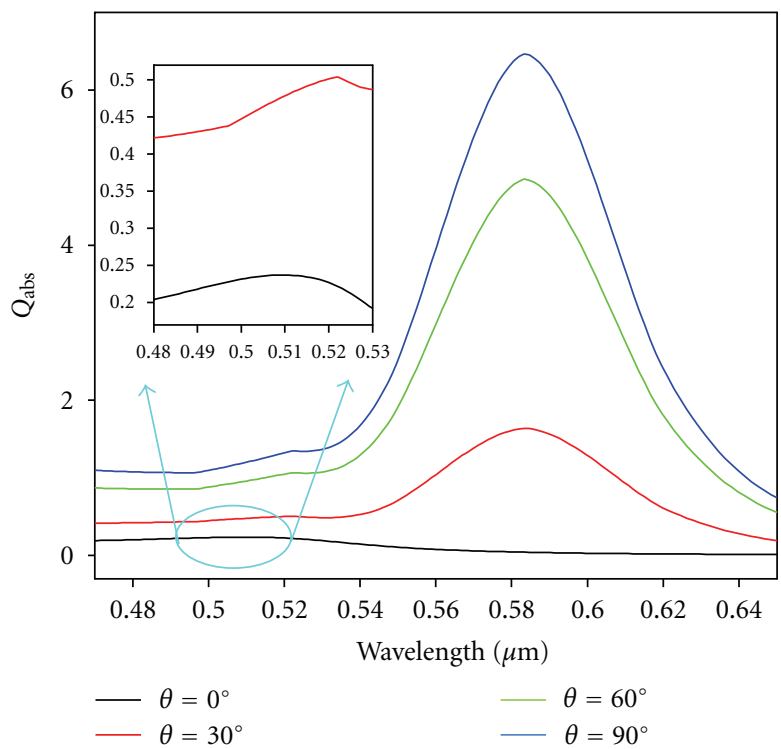

(a)

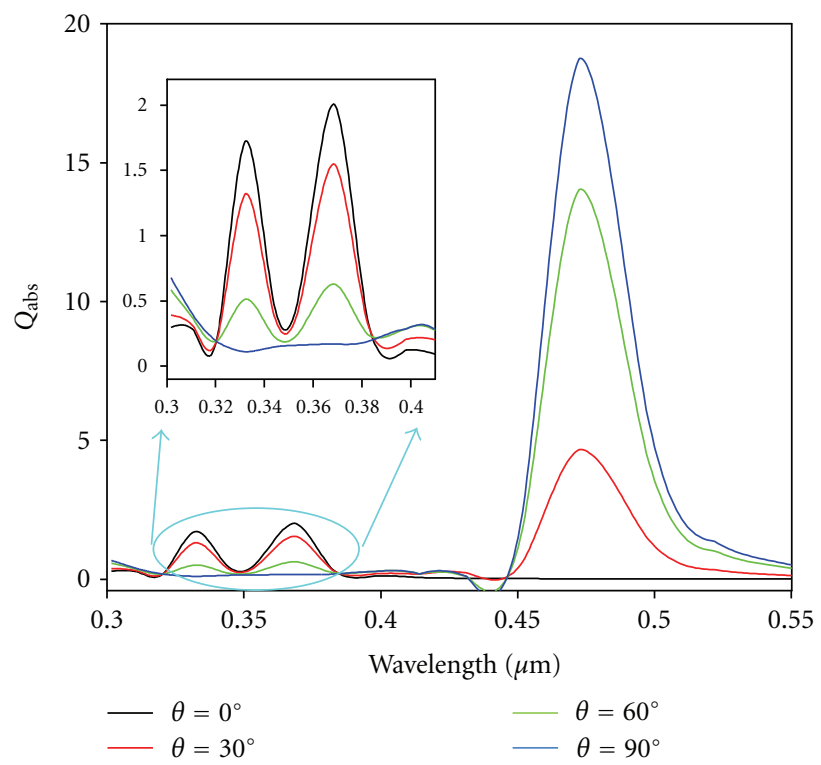

(b)

FIgURE 4: The dependency of the absorption efficiency on the incident angle at a constant rotation angle $\left(\beta=60^{\circ}\right)$ for $(\mathrm{a})$ Au and (b) Ag ellipsoidal nanoparticles. The insets represent the spectra in selected wavelength ranges.

the incident angle, the LM is not observed, and the hybrid TM is predominant. Since the Ag-TMs are well separated, the three plasmonic bands can be observed simultaneously when $15^{\circ} \leq \beta \leq 75^{\circ}$ and $156^{\circ} \leq \theta \leq 75^{\circ}$. The previous observations regarding the dependency of the plasmonic band intensity on the incident angle remain valid for the $\mathrm{Ag}$ ellipsoid.
4.2. Effect of the Length Distribution on the Optical Response of the Ellipsoidal Nanoparticles. To investigate the effect of length distribution on the absorption coefficient, a series of simulation were performed for different lengths of each semiaxis. The dependency of the band position of the dipolar plasmonic modes on the axis length is examined for both $\mathrm{Au}$ and Ag scalene ellipsoidal nanoparticles. First, at fixed length 


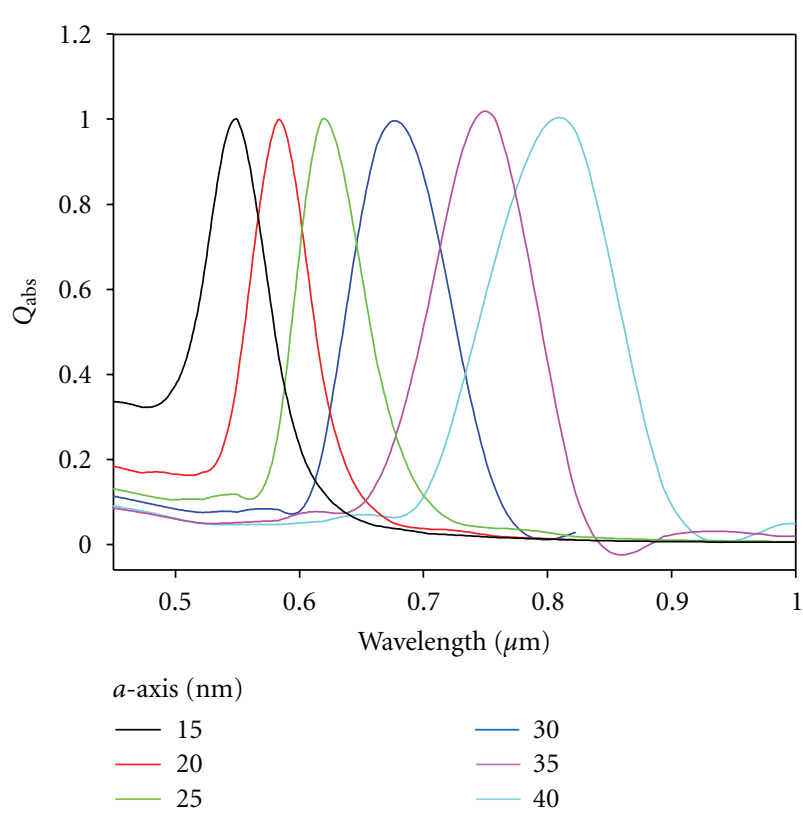

(a)

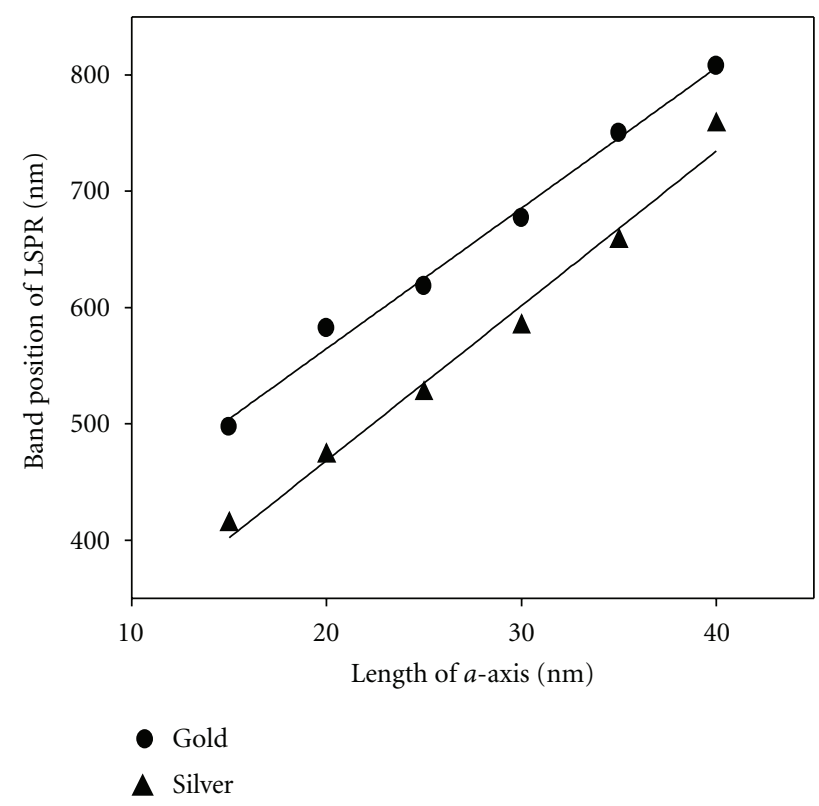

(c)

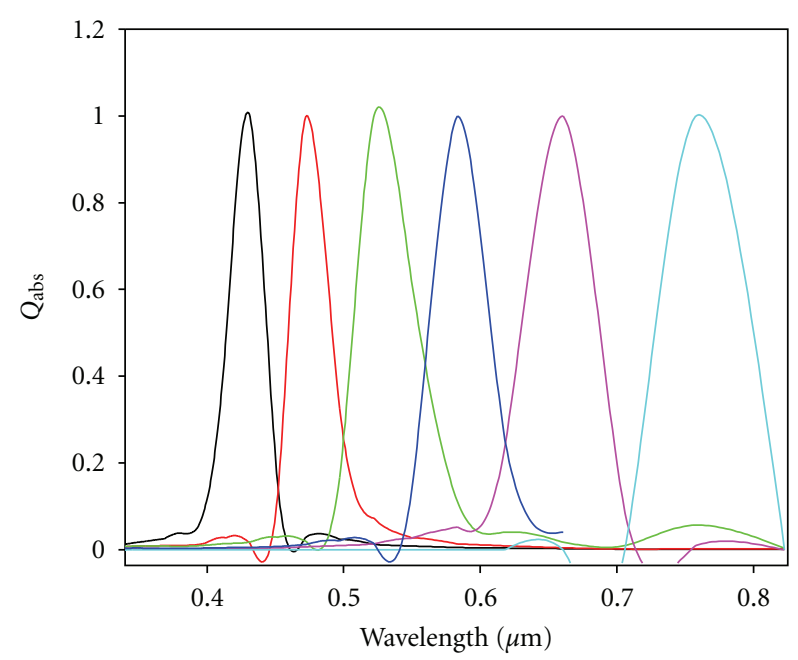

$a$-axis (nm)

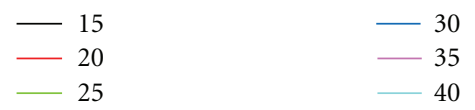

(b)

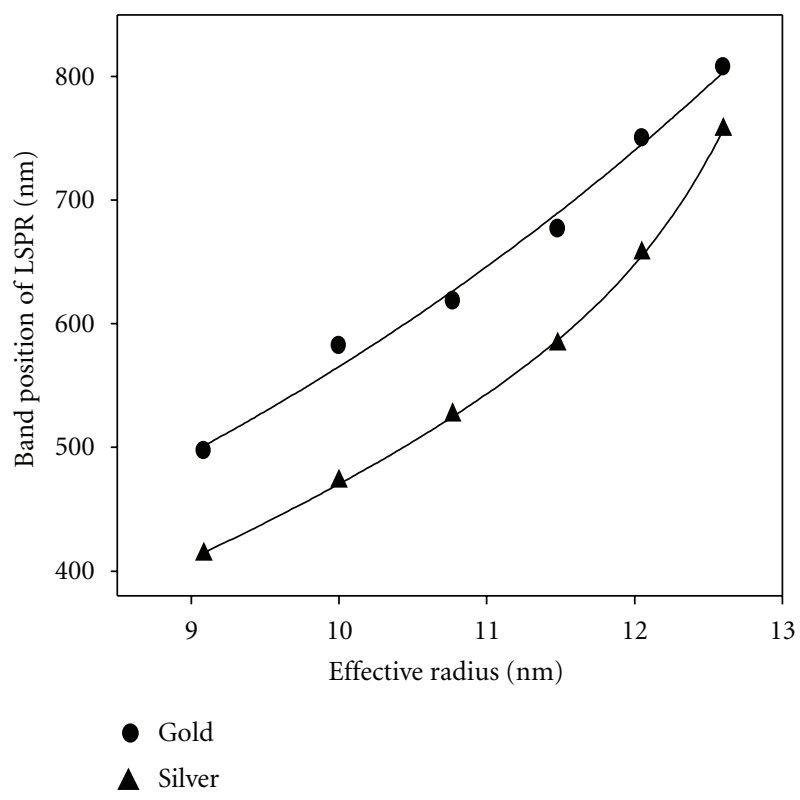

(d)

Figure 5: Normalized absorption spectra of a (a) Au and (b) Ag ellipsoid of different $a$-axis length, (c) the band position of LM versus the length of the $a$-axis. (d) the band position of LM as a function of the nanoellipsoid effective radius. The $b$-axis and the $c$-axis are respectively 10 and $5 \mathrm{~nm}$.

of $b$-axis and $c$-axis with various A.R., the characteristic of the LM is investigated in terms of its length. Secondly, the lengths of the $a$-axis and $c$-axis are kept constant at $50 \mathrm{~nm}$ and $10 \mathrm{~nm}$, respectively, and the $b$-axis length is varied with $b \in\{15,20,25,30,35,40\} \mathrm{nm}$ with a corresponding A.R $=5$. Different $c$-axis lengths with fixed $a, b$-axis values will be the final case. In all cases, the wavelength of either the LM or the TMs will be plotted versus the axis length. As well, the energy of the LSPR band will be presented in terms of the effective size of the selected ellipsoidal particles.
The incident angle is set to be $90^{\circ}$ when the $a$-axis is directed along the $y$-axis. In such a situation, the rotation of the ellipsoidal in the target frame occurs in the $x z$-plane such that $\beta$ does not have any effect on the optical response of the particle. The length of the transverse axes is kept constant, at respectively, $10 \mathrm{~nm}$ ( $b$-axis) and $5 \mathrm{~nm}$ ( $c$-axis) while the $a$ axis is varied in the domain $\in\{15,20,25,30,35,40\} \mathrm{nm}$.

To study the influence of the length distribution on the position of the LM, the simulated absorption is plotted versus the incident wavelength for different lengths as shown in 


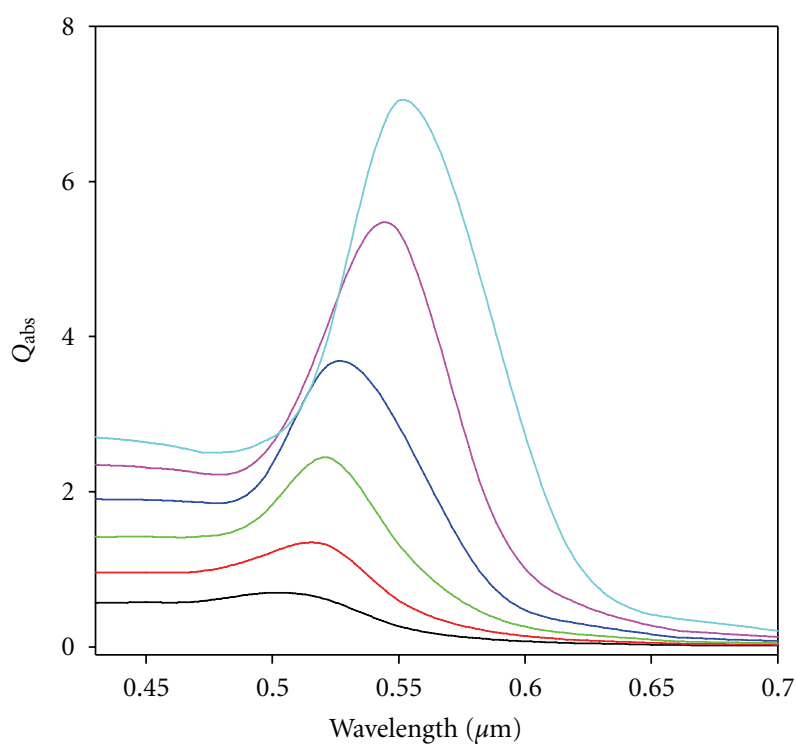

$b$-axis $(\mathrm{nm})$

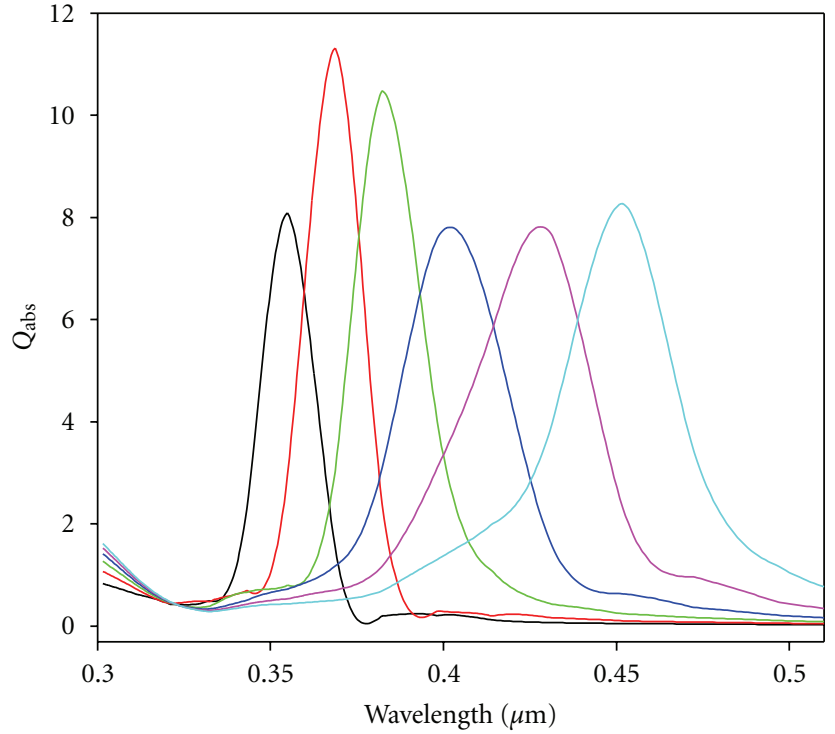

$b$-axis $(\mathrm{nm})$

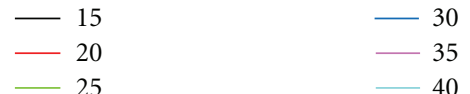

(b)

(a)

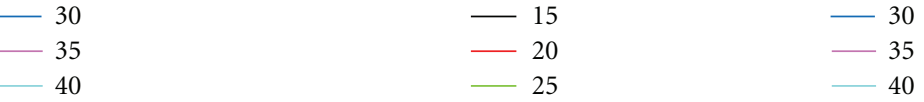

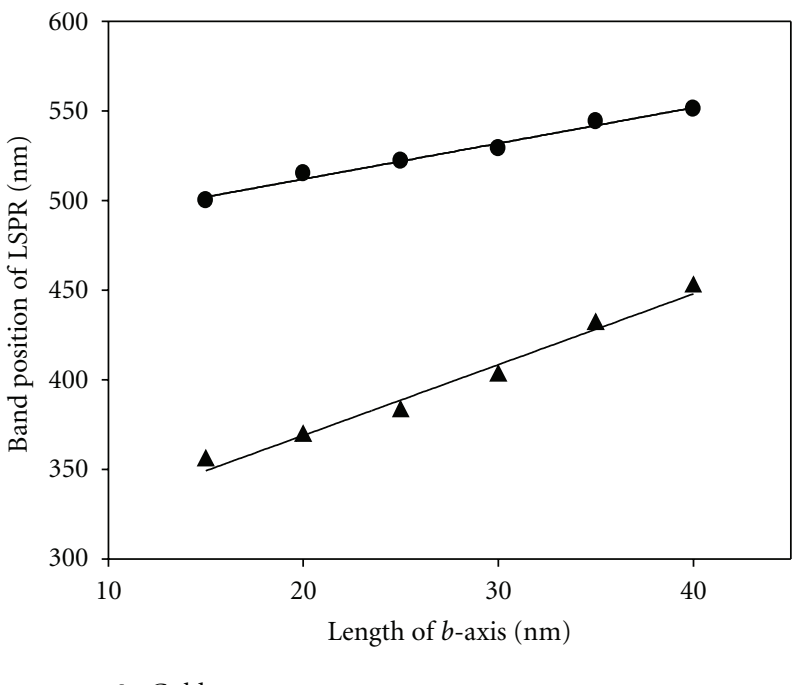

Gold

A Silver

(c)

Figure 6: Absorption efficiency spectra of (a) Au and (b) Ag ellipsoid at different $b$-axis lengths, (c) the band position of LM versus the length of the $b$-axis. The respective values for $a$-axis and $c$-axis are 50 and $10 \mathrm{~nm}$.

Figures 5(a) and 5(b). The position of LM is found to be red shifted, and its intensity is increased with the length (data are shown after normalization). The band position can therefore be tuned in both the visible and near-infrared regions. The $\mathrm{Au}-\mathrm{LM}$ is broader when compared to the corresponding AgLM, and the FWHM is increased with the length in both metallic nanoellipsoids. The change in the longitudinal band position with the length shows a linear variation with a comparable slope as shown in Figure 5(c). The excitation of the Ag-LM occurs at shorter wavelengths as compared to the calculated one for Au. Figure 5(d) shows the modification in the band position of the LM when the effective radius of the ellipsoidal nanoparticle is changed. It seems that the difference in the excited wavelength increases with the length, and it is larger in the case of Ag as compared with the Au's case.

We will now consider the effect of the length distribution of the $b$-axis on the characteristics of the induced charge 


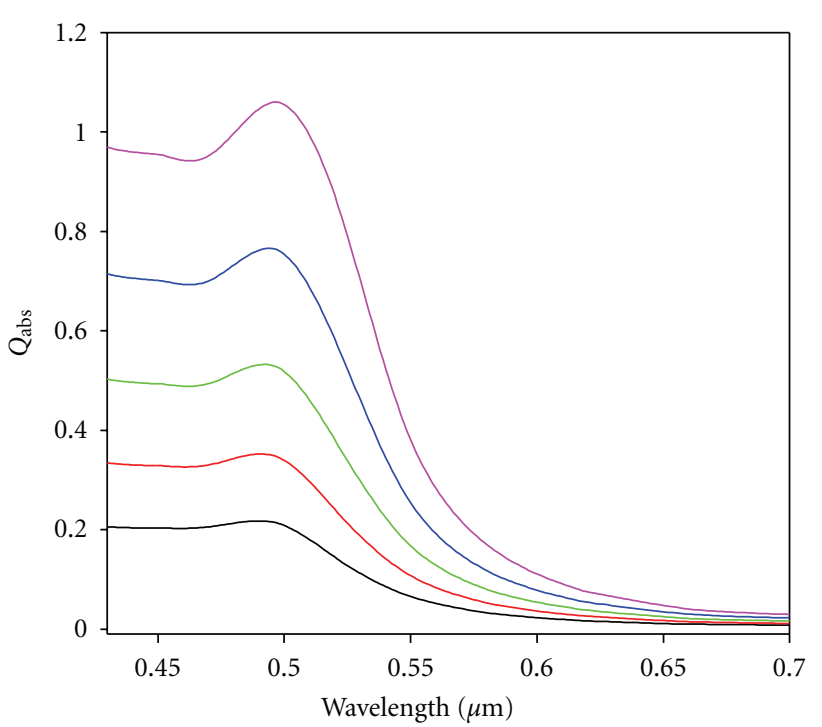

$c$-axis $(\mathrm{nm})$

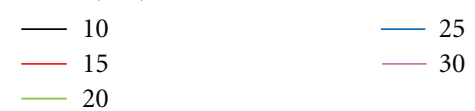

(a)

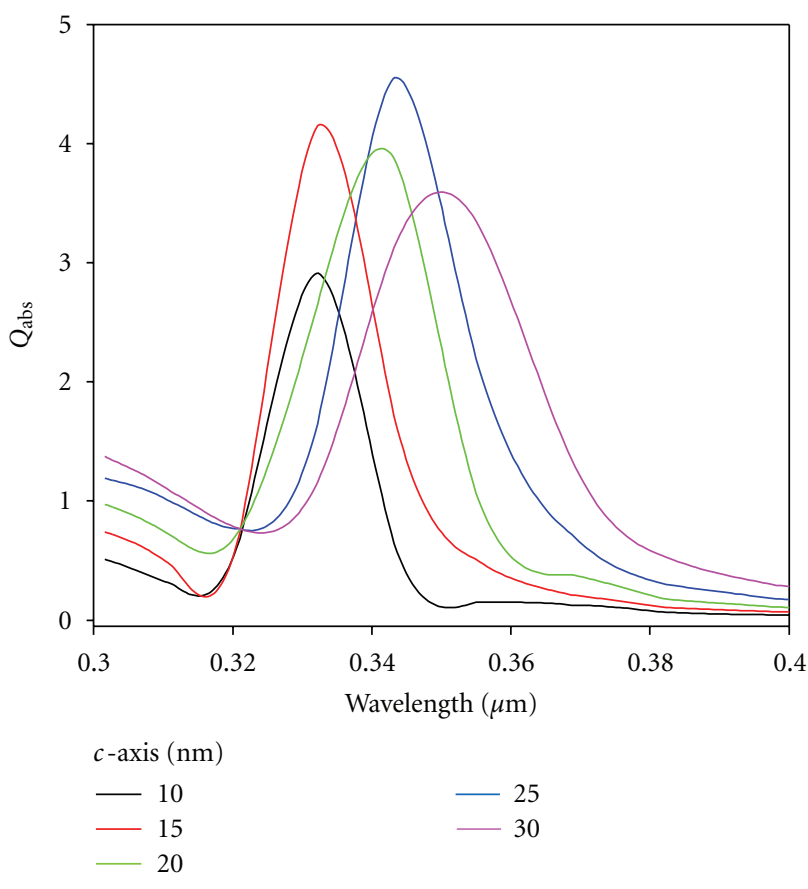

(b)

Figure 7: absorption coefficient of (a) Au and (b) Ag ellipsoid at different $c$-axis lengths. The respective lengths of the $a$-axis and the $b$-axis are 50 and $40 \mathrm{~nm}$.

oscillation along that axis. The target is oriented in the incident electromagnetic field in such a way that the major axis is parallel to the direction of the propagation and the $b$ axis is aligned along the incident electric field by $\left(\beta=0^{\circ}\right)$. The absorption spectra for the Au ellipsoidal nanoparticles at different lengths of $b$-axis are shown in Figure 6(a). It can be noted that the band position of the $b$-TM is red shifted with the length. The change in the excited energy of the plasmonic band is accompanied by a dramatic increase in the absorption amplitude. On the other hand, the optical response of the corresponding $\mathrm{Ag}$ nanoellipsoid is quite different regarding the change in the intensity and the red shift in the band position of the TM. In the latter case, the absorption cross-section contributes mainly to the extinction one in a certain range of length; otherwise, the scattering cross-section is dominant. The slope of the Ag-linear trend is twice compared to the one calculated for $\mathrm{Au}$.

The band position of the TM excited along the $c$-axis for $\mathrm{Au}$ (Figure 7(a)) changes insignificantly with increases in the absorption amplitude while the calculations for Ag show more pronounced changes in both the excited wavelength and the absorption amplitude as shown in Figure $7(\mathrm{~b})$. The band positions of the PMs that correspond to the plasmon oscillations along the $b$ and $c$-axes show less dependency on the length distribution in both metals as compared with the one found for the length distribution of the $a$-axis.

\section{Conclusion}

The optical response of the metallic ellipsoidal nanoparticle is quite specific when compared to other nanoparticle morphologies due to the possibility of three different LSPR modes. These modes are associated with the electronic fluctuations along each symmetry principal axis. The possibility of observing the distinct plasmonic bands depends on the orientation the ellipsoid in both the lab and the target frames. The excitation of the Ag-LSPR modes always occurs at higher energy as compared with the ones calculated for the Au particle. The Ag-PMs are well separated, so that the three modes could be found simultaneously at a given combination of the orientation and rotational angles while the band positions of Au-TMs are very close to each other making them indistinguishable. The band position of the PMs depends linearly on the axis length in both metals. Increase in the axis length results in a red shift of the PM band position. The Ag-PMs are relatively more broadened when compared to the ones found for Au nanoparticles. For both metals, the LM shows a stronger dependency on the length distribution of the axis as compared to the TMs.

The plasmon spectroscopy of a single Au nanoparticle performed by Kalkbrenner et al. [31] has shown three distinct plasmon resonances found in an ellipsoidal particle. The resonance wavelengths were located, respectively, at $614 \mathrm{~nm}$ for the longest axis ( $a$-axis), $571 \mathrm{~nm}$ for the $b$-axis, and $528 \mathrm{~nm}$ for the $c$-axis. This display of a systematic blue shift as the axis becomes shorter is consistent with our calculations, and the range of the shift is also comparable. As mentioned by these authors, the locations of these resonance wavelengths could however have been influenced by the glass tip to which the nanoparticle was attached. Such influence could result in a slight shift in the resonance wavelengths, and in fact, our calculations can be extended to take into account the tip influence. As far as we know, no similar plasmon spectroscopy for a single Ag ellipsoidal particle 
can be found. Considering the fact that the three plasmon resonances in the Ag case are well separated and more easily observable, experiments performed with a single Ag ellipsoidal nanoparticle would be of great interest. Interactions with nearby molecules are expected to modify substantially the resonance wavelengths as well as the bandwidths of the plasmon resonances, and the DDA approach can be effectively used to study these interactions.

\section{Acknowledgments}

This work has been supported by the Natural Sciences and Engineering Research Council of Canada (NSERC) which is gratefully acknowledged. The authors also express their gratitude to B. T. Draine (Princeton University) and P. J. Flatau (University of California) for the free DDA code (DDSCAT 7.1).

\section{References}

[1] K. A. Willets and R. P. Van Duyne, "Localized surface plasmon resonance spectroscopy and sensing," Annual Review of Physical Chemistry, vol. 58, pp. 267-297, 2007.

[2] Y. Xia and N. J. Halas, "Shape-controlled synthesis and surface plasmonic properties of metallic nanoparticles," MRS Bulletin, vol. 30, no. 5, pp. 338-348, 2005.

[3] D. J. Campbell and Y. Xia, "Plasmons: why should we care?" Journal of Chemical Education, vol. 84, no. 1, pp. 91-96, 2007.

[4] J. Henzie, J. Lee, M. H. Lee, W. Hasan, and T. W. Odom, "Nanofabrication of plasmonic structures," Annual Review of Physical Chemistry, vol. 60, pp. 147-165, 2009.

[5] S. Link and M. A. El-Sayed, "Spectral Properties and Relaxation Dynamics of Surface Plasmon Electronic Oscillations in Gold and Silver Nanodots and Nanorods," Journal of Physical Chemistry B, vol. 103, no. 40, pp. 8410-8426, 1999.

[6] C. Noguez, "Surface plasmons on metal nanoparticles: the influence of shape and physical environment," Journal of Physical Chemistry C, vol. 111, no. 10, pp. 3606-3619, 2007.

[7] S. Kim, Y. J. Jung, G. H. Gu, J. S. Suh, S. M. Park, and S. Ryu, "Discrete dipole approximation calculations of optical properties of silver nanorod arrays in porous anodic alumina," Journal of Physical Chemistry C, vol. 113, no. 37, pp. 1632116328, 2009.

[8] P. Yang, H. Portalès, and M. P. Pileni, "Ability to discern the splitting between longitudinal and transverse plasmon resonances in Au compared to Ag nanoparticles in close-packed planar arrays," Physical Review B, vol. 81, no. 20, Article ID 205405, 2010.

[9] H. Portalès, N. Pinna, and M. P. Pileni, "Optical response of ultrafine spherical silver nanoparticles arranged in hexagonal planar arrays studied by the DDA method," Journal of Physical Chemistry A, vol. 113, no. 16, pp. 4094-4099, 2009.

[10] M. Moskovits, "Surface-enhanced Raman spectroscopy: a brief retrospective," Journal of Raman Spectroscopy, vol. 36, no. 6-7, pp. 485-496, 2005.

[11] R. F. Aroca, R. A. Alvarez-Puebla, N. Pieczonka, S. SanchezCortez, and J. V. Garcia-Ramos, "Surface-enhanced Raman scattering on colloidal nanostructures," Advances in Colloid and Interface Science, vol. 116, no. 1-3, pp. 45-61, 2005.

[12] B. Nikoobakht, J. Wang, and M. A. El-Sayed, "Surfaceenhanced Raman scattering of molecules adsorbed on gold nanorods: off-surface plasmon resonance condition," Chemical Physics Letters, vol. 366, no. 1-2, pp. 17-23, 2002.

[13] R. A. Pala, J. White, E. Barnard, J. Liu, and M. L. Brongersma, "Design of plasmonic thin-film solar cells with broadband absorption enhancements," Advanced Materials, vol. 21, no. 34, pp. 3504-3509, 2009.

[14] V. E. Ferry, M. A. Verschuuren, H. B. T. Li et al., "Light trapping in ultrathin plasmonic solar cells," Optics Express, vol. 18, no. 13, pp. A237-A245, 2010.

[15] D. Derkacs, S. H. Lim, P. Matheu, W. Mar, and E. T. Yu, "Improved performance of amorphous silicon solar cells via scattering from surface plasmon polaritons in nearby metallic nanoparticles," Applied Physics Letters, vol. 89, no. 9, Article ID 093103, 2006.

[16] N. Chandrasekharan and P. Y. Kainat, "Improving the photoelectrochemical performance of nanostructured $\mathrm{TiO} 2$ films by adsorption of gold nanoparticles," Journal of Physical Chemistry B, vol. 104, no. 46, pp. 10851-10857, 2000.

[17] V. E. Ferry, L. A. Sweatlock, D. Pacifici, and H. A. Atwater, "Plasmonic nanostructure design for efficient light coupling into solar cells," Nano Letters, vol. 8, no. 12, pp. 4391-4397, 2008.

[18] W. P. McConnell, J. P. Novak, L. C. Brousseau, R. R. Fuierer, R. C. Tenent, and D. L. Feldheim, "Electronic and optical properties of chemically modified metal nanoparticles and molecularly bridged nanoparticle arrays," Journal of Physical Chemistry B, vol. 104, no. 38, pp. 8925-8930, 2000.

[19] W. Cai, T. Gao, H. Hong, and J. Sun, "Applications of gold nanoparticles in cancer nanotechnology," Nanotechnology and Science Applications, vol. 1, pp. 17-32, 2008.

[20] J. Qian, T. Fu, Q. Zhan, and S. He, "Using some nanoparticles as contrast agents for optical bioimaging," IEEE Journal on Selected Topics in Quantum Electronics, vol. 16, no. 3, Article ID 5325886, pp. 672-684, 2010.

[21] Y. Lia, H. J. Schluesener, and S. Xu, "Gold nanoparticle-based biosensors," Gold Bulletin, vol. 43, no. 1, pp. 29-41, 2010.

[22] J. B. Haun, T.-J. Yoon, H. Lee, and R. Weissleder, "Magnetic nanoparticle biosensors," Wiley Interdisciplinary Reviews, vol. 2, no. 3, pp. 291-304, 2010.

[23] T. R. Jensen, G. C. Schatz, and R. P. Van Duyne, "Nanosphere lithography: surface plasmon resonance spectrum of a periodic array of silver nanoparticles by ultraviolet-visible extinction spectroscopy and electrodynamic modeling," Journal of Physical Chemistry B, vol. 103, no. 13, pp. 2394-2401, 1999.

[24] J. C. Hulteen and R. P. van Duyne, "Nanosphere lithography: a materials general fabrication process for periodic particle array surfaces," Journal of Vacuum Science and Technology A, vol. 13, no. 3, pp. 1553-1558, 1995.

[25] Y.-Y. Yu, S.-S. Chang, C.-L. Lee, and C. R. C. Wang, "Gold nanorods: electrochemical synthesis and optical properties," Journal of Physical Chemistry B, vol. 101, no. 34, pp. 66616664, 1997.

[26] M. A. El-Sayed, "Some interesting properties of metals confined in time and nanometer space of different shapes," Accounts of Chemical Research, vol. 34, no. 4, pp. 257-264, 2001.

[27] C. J. Murphy, T. K. Sau, A. M. Gole et al., "Anisotropic metal nanoparticles: synthesis, assembly, and optical applications," Journal of Physical Chemistry B, vol. 109, no. 29, pp. 1385713870, 2005.

[28] P. Sarkar, D. K. Bhui, H. Bar et al., "DDA-Based Simulation of UV-vis Extinction Spectra of Ag Nanorods Synthesized 
Through Seed-Mediated Growth Process," Plasmonics, pp. 19, 2010.

[29] J. Grand, P.-M. Adam, A. S. Grimault et al., "Optical extinction spectroscopy of oblate, prolate and ellipsoid shaped gold nanoparticles: experiments and theory," Plasmonics, vol. 1, no. 2-4, pp. 135-140, 2006.

[30] D. V. Guzatov, V. V. Klimov, and M. Y. Pikhota, "Plasmon oscillations in ellipsoid nanoparticles: beyond dipole approximation," Laser Physics, vol. 20, no. 1, pp. 85-99, 2010.

[31] T. Kalkbrenner, U. Håkanson, and V. Sandoghdar, "Tomographic plasmon spectroscopy of a single gold nanoparticle," Nano Letters, vol. 4, no. 12, pp. 2309-2314, 2004.

[32] B. T. Draine, "The discrete-dipole approximation and its application to interstellar graphite grains," Astrophysical Journal, vol. 333, pp. 848-872, 1988.

[33] B. T. Draine and P. J. Flatau, "Discrete-dipole approximation for scattering calculations," Journal of the Optical Society of America A, vol. 11, no. 4, pp. 1491-1499, 1994.

[34] B. T. Draine and J. Goodman, "Beyond Clausius-Mossotti: wave propagation on a polarizable point lattice and the discrete dipole approximation," Astrophysical Journal, vol. 405, no. 2, pp. 685-697, 1993.

[35] B. T. Draine and P. J. Flatau, "Diserete-dipole approximation for periodic targets: theory and tests," Journal of the Optical Society of America A, vol. 25, no. 11, pp. 2693-2703, 2008.

[36] M. J. Collinge and B. T. Draine, "Discrete-dipole approximation with polarizabilities that account for both finite wavelength and target geometry," Journal of the Optical Society of America A, vol. 21, no. 10, pp. 2023-2028, 2004.

[37] J. J. Goodman, B. T. Draine, and P. J. Flatau, "Application of fast-Fourier-transform techniques to the discrete-dipole approximation," Optics Letters, vol. 16, no. 15, pp. 1198-1200, 1991.

[38] E. M. Purcell and C. R. Pennypacker, "Scattering and absorption of light by nonspherical dielectric grains," Astrophysical Journal, vol. 186, pp. 705-714, 1973.

[39] A. Pinchuk, G. Von Plessen, and U. Kreibig, "Influence of interband electronic transitions on the optical absorption in metallic nanoparticles," Journal of Physics D, vol. 37, no. 22, pp. 3133-3139, 2004. 

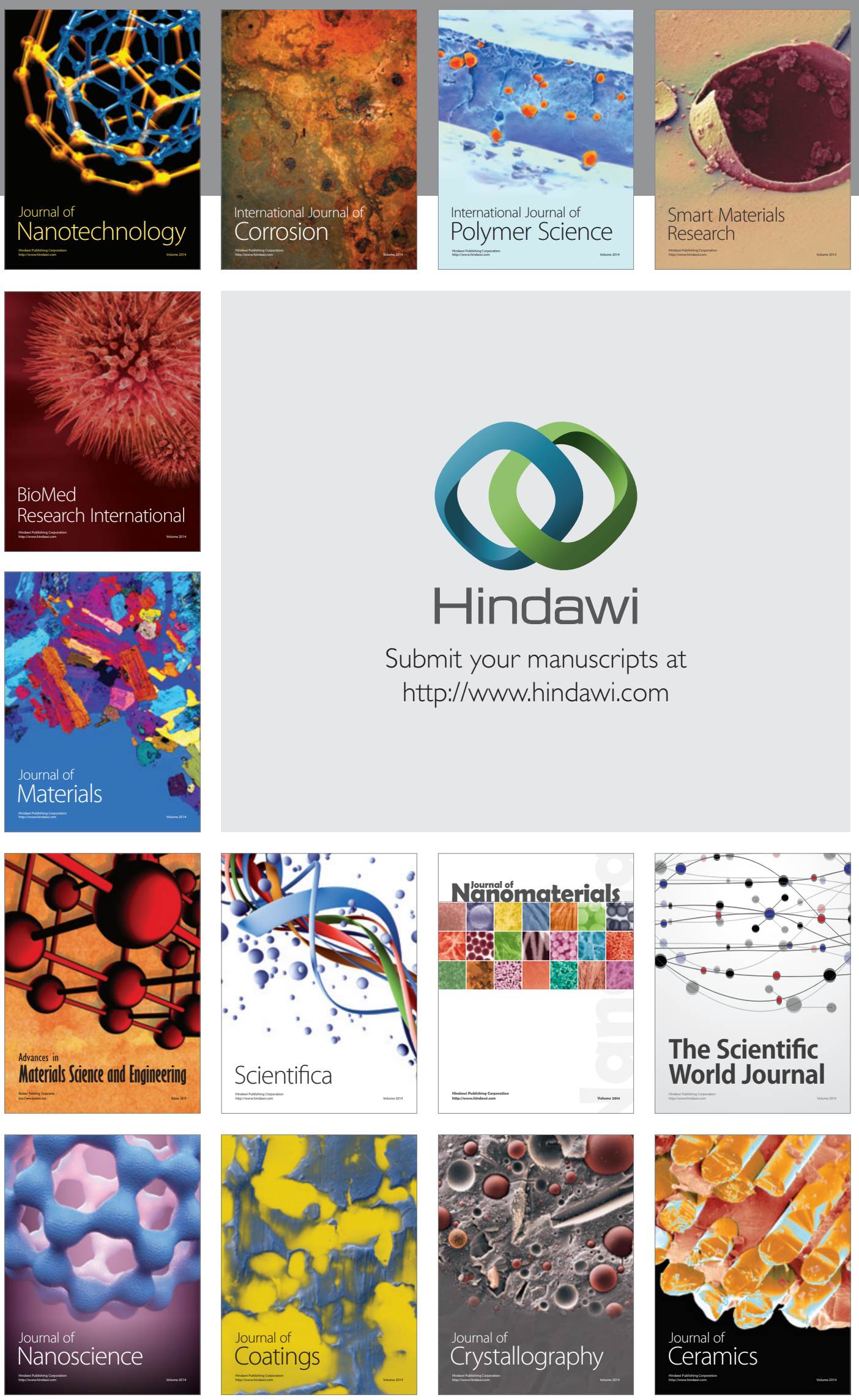

The Scientific World Journal

Submit your manuscripts at

http://www.hindawi.com

\section{World Journal}

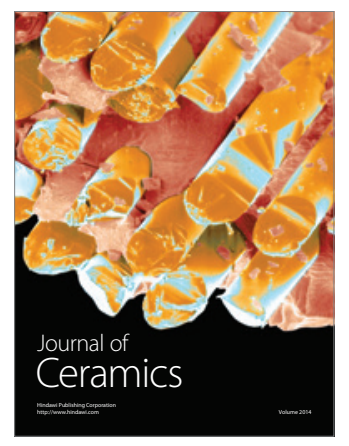

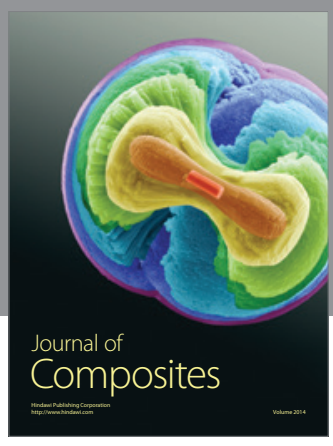
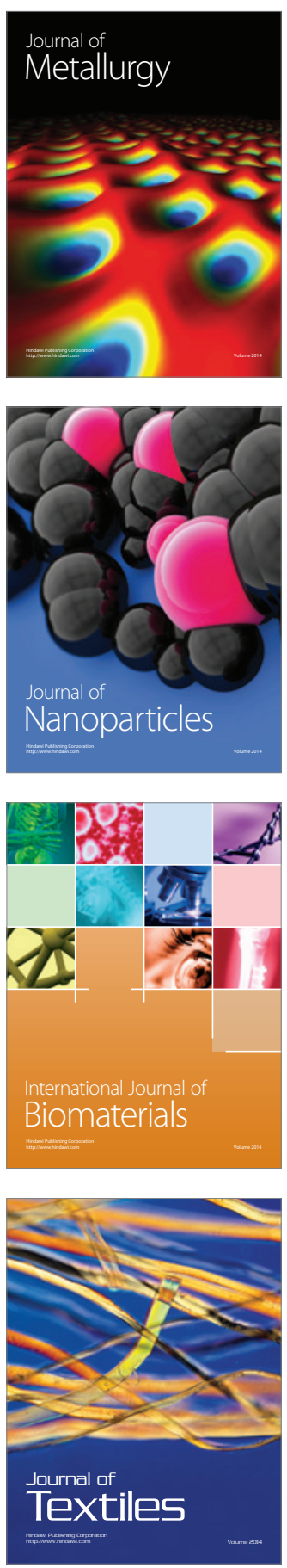